\title{
Non-planar two-loop Feynman integrals contributing to Higgs plus jet production
}

\author{
Hjalte Frellesvig* \\ Institute for Theoretical Particle Physics (TTP), Karlsruhe Institute of Technology \\ Engesserstraße 7, D-76128 Karlsruhe, Germany \\ E-mail: hjalte.frellesvig@kit.edu
}

\section{Roberto Bonciani}

Sapienza - Universita di Roma, Dipartimento di Fisica,

Piazzale Aldo Moro 5, 00185, Rome, Italy, \&

INFN Sezione di Roma,

Piazzale Aldo Moro 2, 00185, Rome, Italy

\section{Vittorio Del Duca}

Institut für theoretische Physik, ETH Zürich,

Wolfgang-Paulistr. 27, 8093 Zürich, Switzerland

\section{Francesco Moriello}

Institut für theoretische Physik, ETH Zürich,

Wolfgang-Paulistr. 27, 8093 Zürich, Switzerland

\section{Johannes Henn}

PRISMA Cluster of Excellence, Johannes Gutenberg University,

55128 Mainz, Germany, \&

Max-Planck-Institute für Physik, Werner-Heisenberg-Insitut,

80805 Müunchen, Germany

\section{Vladimir Smirnov}

Skobeltsyn Institute of Nuclear Physics of Moscow State University,

19992 Moscow, Russia

This is a contribution to the proceedings of the 2018 "Loops and Legs" conference. It is based on a talk by HF on ongoing work on the non-planar Feynman integrals contributing to $H+j$ production at Next-to Leading order in QCD, retaining the complete dependence on the mass of the top-quark. The various non-planar sectors are discussed along with the elliptic structures that appear.

Loops and Legs in Quantum Field Theory

29th of April to 4th of May 2018

St. Goar, Germany

\footnotetext{
${ }^{*}$ Speaker.
} 


\section{Introduction}

Since the 2012 discovery of the Higgs boson [1, 2], investigations into its exact properties and the extent to which they agree with the Standard Model, have been ongoing.

While a fully inclusive cross section for Higgs production is suitable for the measurement of most properties, there are effects that only reveal themselves in the $p_{T}$ distribution. Having the Higgs to be produced with a non-zero $p_{T}$ requires it to be produced together with something else, and at the LHC that is most likely to be a hadronic jet.

As the Higgs boson does not couple directly to (massless) partons, the leading contribution to $H+j$ will be a one-loop process, in which the Higgs couples to a top-quark circulating in the loop. That contribution is known exactly [3,4]. At next-to leading order in QCD that is no longer the case. The NLO contribution is known in the HEFT limit, in which the top-quark is considered infinitely heavy $[5,6,7,8]$. Additionally it is known as expansions around that $[9,10,11]$ and other $[12,13,14,15,16,17]^{1}$ limits, and a numerical result is also available $[18]^{2}$.

A full analytical result has so far been beyond reach. This is due to the presence of two loops and four scales $\left(s=\left(p_{1}+p_{2}\right)^{2}, t=\left(p_{1}+p_{3}\right)^{2}, m_{H}^{2}=\left(p_{1}+p_{2}+p_{3}\right)^{2}\right.$, and $\left.m_{t}^{2}\right)$ in the virtual contribution to the amplitude, which puts this calculation on the edge of what is possible with the current computational technology.

One important step towards a full analytical result, was the 2016 calculation [19] of the planar Feynman integrals contributing to the process. This proceedings contribution is an overview of the progress that has been made so far on the non-planar integrals.

\section{Method}

This section will describe the methods used for the calculation of the planar and non-planar Feynman integrals. The first step is to classify the integrals needed into integral families, which we define as sets of Feynman integrals for which the propagators are a subset of a set of seven propagators defining the family:

$$
I_{a_{1}, \ldots, a_{9}}^{f}=\iint \frac{\mathrm{d}^{d} k_{1}}{i \pi^{d / 2}} \frac{\mathrm{d}^{d} k_{2}}{i \pi^{d / 2}} \frac{P_{f, 8}^{-a_{8}} P_{f, 9}^{-a_{9}}}{P_{f, 1}^{a_{1}} P_{f, 2}^{a_{2}} P_{f, 3}^{a_{3}} P_{f, 4}^{a_{4}} P_{f, 5}^{a_{5}} P_{f, 6}^{a_{6}} P_{f, 7}^{a_{7}}} .
$$

All Feynman integrals needed for the process will then have to be expressed in terms of a minimal set of master integrals for these families, something that is done using publically available programs $[20,21]$ along with private implementations.

Seven integral families contribute to $g g \rightarrow H g$, and to the two quark channels the integrals form a subset thereof. The seven families, that can be seen in fig. 1, are named with letters from A to G. Families A to D contain only planar integrals, that were computed and presented in ref. [19].

The computation of the planar integrals was done using the method of differential equations [22, 23], simplified by the use of canonical forms [24, 25] for the integrals whenever possible. This turned out to be possible for all except eight of the 125 planar master integrals, and these eight are

\footnotetext{
${ }^{1}$ For a discussion of the work of refs. [16, 17], see the talk and proceedings by K. Kudashkin.

${ }^{2}$ For a discussion of the work of ref. [18], see the talk and proceedings by M. Kerner.
} 

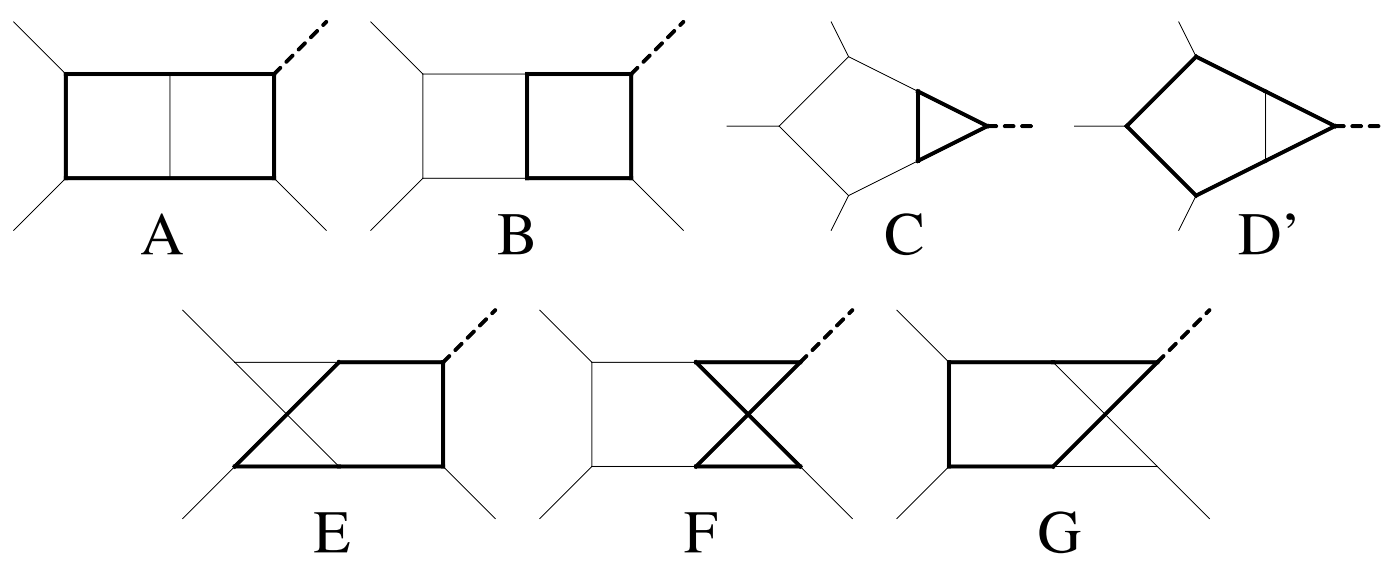

Figure 1: The seven integrals families needed for $g g \rightarrow g H$.

1) A canonical form is available, and a fit to a closed expression can be performed.

2) A canonical form is available, but an integral expression is used at weight 3 and 4 .

3) No canonical form is available, due to the presence of elliptic integrals.

Table 1: The three 'complexity classes' of the integrals contributing to $g g \rightarrow g H$.

integrals that are expressible only in terms of elliptic integrals and integrals thereof, and therefore cannot be brought to canonical form in the traditional sense.

From the canonical differential equation, we extract the 'symbol' $[26,27,28]$ to which we fit an analytical result for the integrals. One additional ingredient that is needed is the values of the integrals in a boundary point, for which we chose $s=t=m_{H}=0$ where most of the canonical master integrals are identically zero.

For many of the integrals we are unable to make a valid fit beyond weight two. So for these cases we express the result at weights three and four as an integral over the lower-weight contributions following ref. [29]. Based on whether or not we can find a canonical form and a closed-form solution, we may split the computed integrals into three 'complexity classes' as can be seen on tab. 1 .

For the planar elliptic cases, a two-fold iterated integral representation was used to express the result.

\section{Non-planar integrals}

Most of the Feynman integrals in the three non-planar integrals families $E, F$, and $G$, will be planar and contained in families $A$ to $D$. Only four genuinely non-planar sectors are present in each family. Of these twelve sectors, two are identical to others under an interchanging of two Mandelstam variables, and this leaves ten genuinely different non-planar sectors, that can be seen listed in tab. 2. Of these ten sectors, seven can be brought completely to canonical form, while the remaining three contain elliptic integrals in one form or the other. It should be noted that the elliptic, non-planar integral denoted as $F_{66}$ is known, and has been computed in ref. [30]. 


\begin{tabular}{|c|c|c|c|}
\hline name & figure & nr. & comment \\
\hline $\mathrm{E}_{60}$ & - & 1 & canonical form \\
\hline $\mathrm{E}_{61}$ & $\mathrm{D}_{63}$ & 2 & canonical form \\
\hline $\mathrm{E}_{63}$ & 4 & canonical form \\
\hline $\mathrm{E}_{67}$ & $\mathrm{Z}_{58}$ & 5 & canonical form \\
\hline $\mathrm{F}_{58}$ & $4 \times 2$ & canonical form \\
\hline
\end{tabular}

\begin{tabular}{|c|c|c|c|}
\hline name & figure & nr. & comment \\
\hline $\mathrm{F}_{66}$ & & 2 & elliptical \\
\hline $\mathrm{F}_{68}$ & & 6 & elliptical $\times 2$ \\
\hline G"63" & & $4 \times 2$ & canonical form \\
\hline G“95”, & & 1 & canonical form \\
\hline G"127" & & 5 & elliptical via $\mathrm{A}_{66}$ \\
\hline
\end{tabular}

Table 2: The ten genuinely different, genuinely non-planar sectors contributing to $g g \rightarrow g H$. "name" refers to the name of the first member of the sector in the internal naming, and "nr." refers to the number of master integrals in the sector. The sectors where the nr. is written as $n \times 2$ are the sectors that appear in two different kinematical channels.

\section{Elliptic sectors}

One aspect that attracted attention to the planar integrals calculated in ref. [19], is the presense of elliptic integrals in the result, as mentioned. To the best of our knowledge, that calculation was the fist time such structures have shown up in a calculation for a genuine scattering (i.e. $2 \rightarrow 2$ ) process. More and more Feynman integrals containing these kinds of structures are being calculated and the mathematical structures therein are being investigated and analyzed, for more recent work, see e.g. refs. [31, 30, 32, 33, 34].

For the planar integrals, there were two elliptic sectors with four integrals in each, both in family A. One is the sector denoted $\mathrm{A}_{66}$ that can be seen drawn in fig. 2. The ellipticity is indicated by the fact that the maximal cut in 4 dimensions, is given by the following integral

$$
\left.\mathrm{A}_{66}\right|_{\max \operatorname{cut}} \propto \int \frac{d z}{s \sqrt{P_{4 ; 1}(z)},}
$$

with

$$
P_{4 ; 1} \equiv\left(\left(m_{H}^{2}+z\right)^{2}-4 m_{H}^{2} m_{t}^{2}\right)\left(4 m_{t}^{2} t u / s+(t+z)^{2}\right),
$$

which will evaluate to a complete elliptic integral of the first kind. For more discussion on the relations between canonical forms, ellipticity, and maximal cuts, see e.g. refs. [25, 35, 36].

Also the planar sector denoted $\mathrm{A}_{70}$ contains elliptic integrals, but there they only enter through the coupling with the $\mathrm{A}_{66}$ sector. The maximal cuts of the integrals in the $\mathrm{A}_{70}$ sector are logarithmic, so only one elliptic curve appears in the planar case, the one defined by the polynomial of eq. (4.2).

In the new, non-planar families, $\mathrm{E}$ is purely canonical and only $\mathrm{G}$ and $\mathrm{F}$ contain elliptic structures. Family G has three elliptic sectors, G"126", G"123", and G"127". G"126" is equivalent to the $\mathrm{A}_{66}$-sector mentioned above, and so is G" 123 " except that is corresponds to a different kinematical 
channel $(t \leftrightarrow u)$, such that

$$
\mathrm{G} \times 123 ” \mid \max \operatorname{cut} \propto \int \frac{d z}{s \sqrt{P_{4 ; 2}(z)},}
$$

with

$$
P_{4 ; 2} \equiv\left(\left(m_{H}^{2}+z\right)^{2}-4 m_{H}^{2} m_{t}^{2}\right)\left(4 m_{t}^{2} t u / s+(u+z)^{2}\right) .
$$

The sector G" 127 " is elliptical through its coupling with the two lower sectors G"126", and G" 123 ", its own maximal cut is logarithmic.
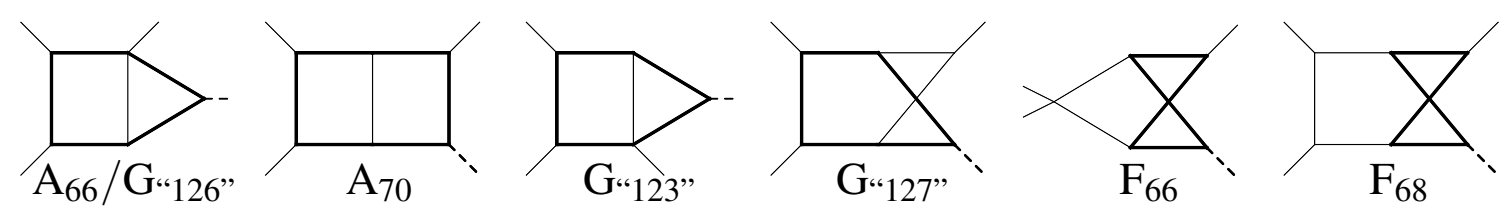

Figure 2: The six elliptic sectors present for $H+j$.

In family F there are two elliptic sectors, both of which are elliptical from their maximal cut

$$
\begin{aligned}
\left.\mathrm{F}_{66}\right|_{\max c u t} & \propto \int \frac{d z}{\left(m_{H}^{2}-t\right) \sqrt{P_{4 ; 3}(z)}}, \\
\left.\mathrm{F}_{68}\right|_{\max c u t} & \propto \int \frac{d z}{t(z+u) \sqrt{P_{4 ; 3}(z)}},
\end{aligned}
$$

with

$$
P_{4 ; 3} \equiv z\left(t-m_{H}^{2}-z\right)\left(4 m_{t}^{2} t-z\left(m_{H}^{2}-t+z\right)\right),
$$

and an additional elliptical contribution to $\mathrm{F}_{68}$ comes from having $\mathrm{F}_{66}$ as a sub-sector.

So we see that three different elliptic curves appear in the non-planar families, as defined by the polynomials $P_{4 ; 1}$ to $P_{4 ; 3}$. And unlike in the planar case there is a sector (G"127") that has contributions from two different elliptic curves, and there is a sector $\left(\mathrm{F}_{68}\right)$ that has the same elliptic curve contributing twice.

Integrating the elliptic sectors using the differential equations, yield results that are so large large that a more desirable method for at least some of these cases should be considered. Such a method could be to abandon the differential equation approach, and instead evaluate the integrals starting from a Feynman parameter representation, as discussed for integrals yielding GPLs in [37] and for certain elliptical cases in [30, 32].

\section{Discussion}

In section 2 we discussed the three different "complexity classes" into which the various integrals may be grouped. Which integrals go into class 3) is given by whether or not they necessarily contain elliptic integrals, something that can be determined with certainty using for instance the 
maximal cut as discussed in section 4. The separation between classes 1) and 2) are not, however, as clear cut. This separation matters, as it determines whether a closed expression in terms of the well-studied function class of Generalized polylogarithms or equivalently $\mathrm{Li}_{n}$ and $\mathrm{Li}_{2,2}$ can be found, or if a one-fold integral representation has to be accepted as the best representation of the result. This is very important for the speed and precision of the numerical evaluation. As it stands, the separation between classes 1) and 2) is determined by whether we are able to find a variable change that allows for the integration of the differential equation system into GPLs, or equivalently if we are able to find a basis of such functions to fit the symbol obtained from the differential equation. To find a definitive mathematical criterion to determine weather or not a differential equation in canonical form can be integrated up to Generalized polylogarithms, would be a interesting endeavor from both a mathematical and practical point of view.

As mentioned in the introduction, obtaining expressions for the integrals discussed in this proceedings contribution, is the biggest challenge towards the computation of the NLO QCD contribution to $H+j$ production at hadron colliders, but it is not the only one. Once the integrals are computed, they will need to be analytically continued to the physical regions of parameter space. Additionally the IR subtraction terms will have to be computed and subtracted before the NLO cross section can be obtained.

\section{References}

[1] G. Aad et al., "Observation of a new particle in the search for the Standard Model Higgs boson with the ATLAS detector at the LHC," Phys. Lett., vol. B716, pp. 1-29, 2012. [arXiv:1207.7214 [hep-ex]]

[2] S. Chatrchyan et al., "Observation of a new boson at a mass of $125 \mathrm{GeV}$ with the CMS experiment at the LHC," Phys. Lett., vol. B716, pp. 30-61, 2012. [arXiv:1207.7235 [hep-ex]].

[3] R. K. Ellis, I. Hinchliffe, M. Soldate, and J. J. Van Der Bij, "Higgs decay to $\pi^{+} \pi^{-}$. A possible signature of intermediate mass Higgs bosons at high energy hadron colliders," Nuclear Physics B, vol. 297, pp. 221-243, Feb. 1988.

[4] U. Baur and E. W. N. Glover, "Higgs Boson Production at Large Transverse Momentum in Hadronic Collisions," Nucl. Phys., vol. B339, pp. 38-66, 1990.

[5] R. Boughezal, F. Caola, K. Melnikov, F. Petriello, and M. Schulze, "Higgs boson production in association with a jet at next-to-next-to-leading order in perturbative QCD," JHEP, vol. 06, p. 072, 2013. [arXiv:1504.07922]

[6] X. Chen, T. Gehrmann, E. W. N. Glover, and M. Jaquier, "Precise QCD predictions for the production of Higgs + jet final states," Phys. Lett., vol. B740, pp. 147-150, 2015. [arXiv:1408.5325]

[7] R. Boughezal, F. Caola, K. Melnikov, F. Petriello, and M. Schulze, "Higgs boson production in association with a jet at next-to-next-to-leading order," Phys. Rev. Lett., vol. 115, no. 8, p. 082003, 2015. [arXiv:1504.07922]

[8] R. Boughezal, C. Focke, W. Giele, X. Liu, and F. Petriello, "Higgs boson production in association with a jet at NNLO using jettiness subtraction," Phys. Lett., vol. B748, pp. 5-8, 2015. [arXiv:1505.03893]

[9] R. V. Harlander, T. Neumann, K. J. Ozeren, and M. Wiesemann, "Top-mass effects in differential Higgs production through gluon fusion at order $\alpha_{s}^{4}$, , JHEP, vol. 08, p. 139, 2012. [arXiv:1206.0157] 
[10] T. Neumann and M. Wiesemann, "Finite top-mass effects in gluon-induced Higgs production with a jet-veto at NNLO,” JHEP, vol. 11, p. 150, 2014. [arXiv:1408.6836]

[11] T. Neumann and C. Williams, "The Higgs boson at high $p_{T}$," Phys. Rev., vol. D95, no. 1, p. 014004, 2017. [arXiv:1609.00367]

[12] R. Mueller and D. G. Öztürk, "On the computation of finite bottom-quark mass effects in Higgs boson production,” JHEP, vol. 08, p. 055, 2016. [arXiv:1512.08570]

[13] K. Melnikov, L. Tancredi, and C. Wever, "Two-loop $g g \rightarrow H g$ amplitude mediated by a nearly massless quark," JHEP, vol. 11, p. 104, 2016. [arXiv:1702.00426]

[14] K. Melnikov, L. Tancredi, and C. Wever, "Two-loop amplitudes for $q g \rightarrow H q$ and $q \bar{q} \rightarrow H g$ mediated by a nearly massless quark,” Phys. Rev., vol. D95, no. 5, p. 054012, 2017. [arXiv:1712.06549]

[15] J. M. Lindert, K. Melnikov, L. Tancredi, and C. Wever, "Top-bottom interference effects in Higgs plus jet production at the LHC," Phys. Rev. Lett., vol. 118, no. 25, p. 252002, 2017. [arXiv:1703.03886]

[16] K. Kudashkin, K. Melnikov, and C. Wever, "Two-loop amplitudes for processes $g g \rightarrow H g, q g \rightarrow H q$ and $q \bar{q} \rightarrow H g$ at large Higgs transverse momentum," JHEP, vol. 02, p. 135, 2018. [arXiv:1712.06549]

[17] J. M. Lindert, K. Kudashkin, K. Melnikov, and C. Wever, "Higgs bosons with large transverse momentum at the LHC," Phys. Lett., vol. B782, pp. 210-214, 2018. [arXiv:1801.08226]

[18] S. P. Jones, M. Kerner, and G. Luisoni, "Next-to-Leading-Order QCD Corrections to Higgs Boson Plus Jet Production with Full Top-Quark Mass Dependence,” Phys. Rev. Lett., vol. 120, no. 16, p. 162001, 2018. [arXiv:1802.00349]

[19] R. Bonciani, V. Del Duca, H. Frellesvig, J. M. Henn, F. Moriello, and V. A. Smirnov, “Two-loop planar master integrals for Higgs $\rightarrow 3$ partons with full heavy-quark mass dependence," JHEP, vol. 12, p. 096, 2016. [arXiv:1609.06685]

[20] A. V. Smirnov, "FIRE5: a C++ implementation of Feynman Integral REduction,” Comput. Phys. Commun., vol. 189, pp. 182-191, 2015. [arXiv:1408.2372]

[21] P. Maierhöfer, J. Usovitsch, and P. Uwer, "Kira - A Feynman integral reduction program," Comput. Phys. Commun., vol. 230, pp. 99-112, 2018. [arXiv:1705.05610]

[22] A. V. Kotikov, "Differential equations method: New technique for massive Feynman diagrams calculation,” Phys. Lett., vol. B254, pp. 158-164, 1991.

[23] T. Gehrmann and E. Remiddi, "Differential equations for two loop four point functions," Nucl. Phys., vol. B580, pp. 485-518, 2000. [arXiv:hep-ph/9912329]

[24] J. M. Henn, "Multiloop integrals in dimensional regularization made simple," Phys. Rev. Lett., vol. 110, p. 251601, 2013. [arXiv:1304.1806]

[25] J. M. Henn, "Lectures on differential equations for Feynman integrals," J. Phys., vol. A48, p. 153001, 2015. [arXiv:1412.2296]

[26] A. B. Goncharov, M. Spradlin, C. Vergu, and A. Volovich, "Classical Polylogarithms for Amplitudes and Wilson Loops,” Phys. Rev. Lett., vol. 105, p. 151605, 2010. [arXiv:1006.5703]

[27] C. Duhr, H. Gangl, and J. R. Rhodes, "From polygons and symbols to polylogarithmic functions," Journal of High Energy Physics, vol. 10, p. 75, Oct. 2012. [arXiv:1110.0458]

[28] C. Duhr, "Hopf algebras, coproducts and symbols: an application to Higgs boson amplitudes," JHEP, vol. 08, p. 043, 2012. [arXiv:1203.0454] 
[29] S. Caron-Huot and J. M. Henn, "Iterative structure of finite loop integrals," JHEP, vol. 06, p. 114, 2014. [arXiv:1404.2922]

[30] M. Hidding and F. Moriello, "All orders structure and efficient computation of linearly reducible elliptic Feynman integrals,” 2017. [arXiv:1712.04441]

[31] J. Broedel, C. Duhr, F. Dulat, and L. Tancredi, "Elliptic polylogarithms and iterated integrals on elliptic curves. Part I: general formalism,” JHEP, vol. 05, p. 093, 2018. [arXiv:1712.07089]

[32] J. L. Bourjaily, A. J. McLeod, M. Spradlin, M. von Hippel, and M. Wilhelm, "Elliptic Double-Box Integrals: Massless Scattering Amplitudes beyond Polylogarithms," Phys. Rev. Lett., vol. 120, no. 12, p. 121603, 2018. [arXiv:1712.02785]

[33] J. Ablinger, J. Blümlein, A. De Freitas, M. van Hoeij, E. Imamoglu, C. G. Raab, C. S. Radu, and C. Schneider, "Iterated Elliptic and Hypergeometric Integrals for Feynman Diagrams," J. Math. Phys., vol. 59, no. 6, p. 062305, 2018. [arXiv:1706.01299]

[34] L. Adams, E. Chaubey, and S. Weinzierl, "Analytic results for the planar double box integral relevant to top-pair production with a closed top loop,” 2018. [arXiv:1806.04981]

[35] A. Primo and L. Tancredi, "Maximal cuts and differential equations for Feynman integrals. An application to the three-loop massive banana graph," Nucl. Phys., vol. B921, pp. 316-356, 2017. [arXiv:1704.05465]

[36] H. Frellesvig and C. G. Papadopoulos, "Cuts of Feynman Integrals in Baikov representation,” JHEP, vol. 04, p. 083, 2017. [arXiv:1701.07356]

[37] E. Panzer, "Algorithms for the symbolic integration of hyperlogarithms with applications to Feynman integrals,” Comput. Phys. Commun., vol. 188, pp. 148-166, 2014. [arXiv:1403.3385] 